PROCEEDINGS OF THE

AMERICAN MATHEMATICAL SOCIETY

Volume 130, Number 6, Pages 1689-1697

S 0002-9939(01)06245-1

Article electronically published on October 17, 2001

\title{
ON ONE PROBLEM OF UNIQUENESS \\ OF MEROMORPHIC FUNCTIONS CONCERNING SMALL FUNCTIONS
}

\author{
HONG-XUN YI
}

(Communicated by Juha M. Heinonen)

\begin{abstract}
In this paper, we show that if two non-constant meromorphic functions $f$ and $g$ satisfy $\bar{E}\left(a_{j}, k, f\right)=\bar{E}\left(a_{j}, k, g\right)$ for $j=1,2, \ldots, 5$, where $a_{j}$ are five distinct small functions with respect to $f$ and $g$, and $k$ is a positive integer or $\infty$ with $k \geq 14$, then $f \equiv g$. As a special case this also answers the longstanding problem on uniqueness of meromorphic functions concerning small functions.
\end{abstract}

\section{INTRODUCTION AND MAIN RESULT}

In this paper, by meromorphic function we shall always mean a meromorphic function in the complex plane $\mathbb{C}$. We adopt the standard notations in the Nevanlinna theory of meromorphic functions as explained in [1]. For any non-constant meromorphic function $f(z)$, we denote by $S(r, f)$ any quantity satisfying

$$
S(r, f)=o(T(r, f))
$$

for $r \rightarrow \infty$ except possibly a set of $r$ of finite linear measure. A meromorphic function $a(z)$ is called a small function with respect to $f(z)$ if $T(r, a)=S(r, f)$. Let $S(f)$ be the set of meromorphic functions in the complex plane $\mathbb{C}$ which are small functions with respect to $f$. Note that $\mathbb{C} \in S(f)$ and $S(f)$ is a field (see [2]).

If $f(z)$ is a non-constant meromorphic function, $a(z) \in S(f) \cup\{\infty\}$, and $k$ is a positive integer or $\infty$, we denote by $\bar{E}(a, k, f)$ the set of distinct zeros of $f(z)-a(z)$ with multiplicities $\leq k$, where $f(z)-\infty$ means $1 / f(z)$ (see [10 p.195]). In particular, we denote by $\bar{E}(a, \infty, f)$ the set of distinct zeros of $f(z)-a(z)$, and we denote it simply by $\bar{E}(a, f)$.

Let $f(z)$ and $g(z)$ be non-constant meromorphic functions and let $a(z) \in\{S(f) \cap$ $S(g)\} \cup\{\infty\}$. We denote by $\bar{N}_{0}(r, a, f, g)$ the counting function of common zeros of $f(z)-a(z)=0$ and $g(z)-a(z)=0$ (ignoring multiplicities), each point counted only once. Let

$$
\bar{N}_{12}(r, a, f, g):=\bar{N}(r, a, f)+\bar{N}(r, a, g)-2 \bar{N}_{0}(r, a, f, g) .
$$

Received by the editors September 22, 2000 and, in revised form, December 1, 2000. 2000 Mathematics Subject Classification. Primary 30D35; Secondary 30D30.

Key words and phrases. Meromorphic function, small function, uniqueness theorem.

This work was supported by the NSFC (NO. 19871050) and the RFDP (No. 98042209). 
Then $\bar{N}_{12}(r, a, f, g)$ denotes the counting function of different solutions to $f(z)-$ $a(z)=0$ and $g(z)-a(z)=0$ (see [7, p.107]). If

$$
\bar{N}(r, a, f)-\bar{N}_{0}(r, a, f, g)=0 \quad \text { and } \quad \bar{N}(r, a, g)-\bar{N}_{0}(r, a, f, g)=0,
$$

we say $f(z)$ and $g(z)$ share $a(z)$ IM. If

$$
\bar{N}(r, a, f)-\bar{N}_{0}(r, a, f, g)=S(r, f) \quad \text { and } \quad \bar{N}(r, a, g)-\bar{N}_{0}(r, a, f, g)=S(r, g),
$$

we say $f(z)$ and $g(z)$ share $a(z)$ "IM" (see [10, p.254]). It is obvious that if $f(z)$ and $g(z)$ share $a(z) \mathbf{I M}$, then $\bar{E}(a, f)=\bar{E}(a, g)$, and $\bar{N}_{12}(r, a, f, g)=0$; if $f(z)$ and $g(z)$ share $a(z)$ "IM", then $\bar{N}_{12}(r, a, f, g)=S(r, f)+S(r, g)$.

In the 1920s, R. Nevanlinna established the following famous second fundamental theorem:

Theorem A ([7, p.70]). Let $f(z)$ be a non-constant meromorphic function, and let $a_{1}, a_{2}, \cdots, a_{q}$ be $q(\geq 3)$ distinct elements in $\mathbb{C} \cup\{\infty\}$. Then

$$
(q-2) T(r, f) \leq \sum_{j=1}^{q} \bar{N}\left(r, a_{j}, f\right)+S(r, f) .
$$

In 1929, R. Nevanlinna proved the following well-known theorem on the uniqueness of meromorphic functions by making use of Theorem A:

Theorem B ([7, p.109], see also [1, p.48]). If $f$ and $g$ are meromorphic functions sharing $a_{j} \mathbf{I M}$ for $j=1,2, \ldots, 5$, where $a_{1}, a_{2}, \ldots, a_{5}$ are five distinct elements in $\mathbb{C} \cup\{\infty\}$, then $f \equiv g$.

In [7, p.111], R. Nevanlinna proved that Theorem B is sharp. It is natural to ask the following problem, which is the long-standing one:

Problem A (see [7, p.77 and p.109], see also [2]-[6], 8]-[11]). Does Theorem B hold if $a_{1}, a_{2}, \ldots, a_{5}$ are five distinct elements in $\{S(f) \cap S(g)\} \cup\{\infty\}$ ?

In recent years, some partial results were obtained on Problem A (see 2]-6], [8]-[11]). In this paper, we give a positive answer to Problem A. In fact, we prove more generally the following theorem:

Theorem 1. Let $f$ and $g$ be non-constant meromorphic functions and let $a_{j} \quad(j=$ $1,2, \ldots, 5)$ be five distinct elements in $\{S(f) \cap S(g)\} \cup\{\infty\}$. If

$$
\bar{E}\left(a_{j}, k, f\right)=\bar{E}\left(a_{j}, k, g\right) \quad(j=1,2, \ldots, 5),
$$

where $k$ is a positive integer or $\infty$ with $k \geq 14$, then $f \equiv g$.

By Theorem 1, we obtain the following corollary:

Corollary 1. Let $f$ and $g$ be non-constant meromorphic functions and let $a_{j} \quad(j=$ $1,2, \ldots, 5)$ be five distinct elements in $\{S(f) \cap S(g)\} \cup\{\infty\}$. If $f$ and $g$ share $a_{j}$ IM $(j=1,2, \ldots, 5)$, then $f \equiv g$.

It is obvious that Corollary 1 answers the above Problem A in the affirmative. 


\section{Some Lemmas}

Lemma 1 ([11], see also [2, Lemma 2] or [10, p.185]). Let $f(z)$ be a non-constant meromorphic function and let $a_{1}, a_{2}, \ldots, a_{5}$ be five distinct elements in $S(f) \cup\{\infty\}$. Then

$$
2 T(r, f) \leq \sum_{j=1}^{5} \bar{N}\left(r, a_{j}, f\right)+S(r, f) .
$$

Let $f(z)$ be a non-constant meromorphic function, $a(z) \in S(f) \cup\{\infty\}$, and $k$ be a positive integer. We denote by $\bar{N}_{k)}(r, a, f)$ the counting function of distinct zeros of $f(z)-a(z)$ with multiplicities $\leq k$, by $\bar{N}_{(k+1}(r, a, f)$ the counting function of distinct zeros of $f(z)-a(z)$ with multiplicities $\geq k+1$, each point in these counting functions counted only once (see [10, p.190]).

Lemma 2. Let $f(z)$ be a non-constant meromorphic function and let $a_{1}, a_{2}, \ldots, a_{5}$ be five distinct elements in $S(f) \cup\{\infty\}$, and $k$ a positive integer. Then

$$
\sum_{j=1}^{5} \bar{N}_{(k+1}\left(r, a_{j}, f\right) \leq \frac{3}{k} T(r, f)+S(r, f) .
$$

Proof. By Lemma 1, we can obtain (2.1). Noting that for $j=1,2, \ldots, 5$

$$
k \bar{N}_{(k+1}\left(r, a_{j}, f\right)+\bar{N}\left(r, a_{j}, f\right) \leq N\left(r, a_{j}, f\right) \leq T(r, f)+S(r, f),
$$

we have from $(2.1)$

$$
k \sum_{j=1}^{5} \bar{N}_{(k+1}\left(r, a_{j}, f\right)+2 T(r, f) \leq 5 T(r, f)+S(r, f) .
$$

From this we get (2.2).

Lemma 3. Let $h$ be a non-constant meromorphic function, and let $a \in S(h)$ and $a \not \equiv 0$. Then

$$
\begin{aligned}
& m\left(r, \frac{a^{\prime} h-a h^{\prime}}{h-a}\right)=S(r, h), \\
& m\left(r, \frac{a^{\prime} h-a h^{\prime}}{h(h-a)}\right)=S(r, h) .
\end{aligned}
$$

Proof. Noting

$$
\begin{aligned}
& \frac{a^{\prime} h-a h^{\prime}}{h-a}=a^{\prime}-\frac{a\left(h^{\prime}-a^{\prime}\right)}{h-a}, \\
& \frac{a^{\prime} h-a h^{\prime}}{h(h-a)}=\frac{h^{\prime}}{h}-\frac{h^{\prime}-a^{\prime}}{h-a},
\end{aligned}
$$

and the lemma of the logarithmic derivative, we obtain Lemma 3.

The following lemma plays an important role in the proof of Theorem 1.

Lemma 4. Let $f$ and $g$ be non-constant meromorphic functions and let $a_{1}, a_{2}, \ldots$, $a_{5}$ be five distinct elements in $\{S(f) \cap S(g)\} \cup\{\infty\}$. If $f \not \equiv g$, then

$$
\bar{N}_{0}\left(r, a_{5}, f, g\right) \leq \sum_{j=1}^{4} \bar{N}_{12}\left(r, a_{j}, f, g\right)+S(r, f)+S(r, g) .
$$




\section{Proof of Lemma 4}

If $\bar{N}_{0}\left(r, a_{5}, f, g\right)=S(r, f)+S(r, g),(2.7)$ obviously holds. In the following we suppose

$$
\bar{N}_{0}\left(r, a_{5}, f, g\right) \neq S(r, f)+S(r, g) .
$$

Set

$$
L(w):=\frac{\left(w-a_{1}\right)\left(a_{3}-a_{2}\right)}{\left(w-a_{2}\right)\left(a_{3}-a_{1}\right)} .
$$

Let $F(z):=L(f(z)), G(z):=L(g(z)), b_{j}:=L\left(a_{j}\right) \quad(j=1,2, \ldots, 5)$. From (3.2) we have $b_{1}=0, b_{2}=\infty, b_{3}=1$,

$$
T(r, F)=T(r, f)+S(r, f), \quad T(r, G)=T(r, g)+S(r, g) .
$$

Since $a_{1}, a_{2}, \ldots, a_{5}$ are five distinct elements in $\{S(f) \cap S(g)\} \cup\{\infty\}$, from (3.2) we know that $b_{1}, b_{2}, \ldots, b_{5}$ are five distinct elements in $\{S(F) \cap S(G)\} \cup\{\infty\}$. Thus, $b_{4}, b_{5} \not \equiv 0,1, \infty$ and $b_{4} \not \equiv b_{5}$. Noting $f \not \equiv g$, we have

$$
F \not \equiv G \text {. }
$$

From (3.1) and (3.3), we get

$$
\bar{N}_{0}\left(r, b_{5}, F, G\right) \neq S(r, F)+S(r, G) .
$$

Set

$$
H:=\frac{F^{\prime}\left(a^{\prime} G-a G^{\prime}\right)(F-G)}{F(F-1) G(G-a)}-\frac{G^{\prime}\left(a^{\prime} F-a F^{\prime}\right)(F-G)}{G(G-1) F(F-a)},
$$

where $a=b_{4}(\neq 0,1)$. Then we have from $(3.6)$

$$
H=\frac{(F-G) Q}{F(F-1)(F-a) G(G-1)(G-a)},
$$

where

$$
Q=F^{\prime}\left(a^{\prime} G-a G^{\prime}\right)(G-1)(F-a)-G^{\prime}\left(a^{\prime} F-a F^{\prime}\right)(F-1)(G-a) .
$$

By a simple computation,

$$
\begin{aligned}
Q= & a^{\prime} F F^{\prime} G^{2}-a^{\prime} F F^{\prime} G-a(a-1) F F^{\prime} G^{\prime}-a a^{\prime} F^{\prime} G^{2}+a a^{\prime} F^{\prime} G \\
& -a^{\prime} F^{2} G G^{\prime}+a^{\prime} F G G^{\prime}+a(a-1) F^{\prime} G G^{\prime}+a a^{\prime} F^{2} G^{\prime}-a a^{\prime} F G^{\prime} .
\end{aligned}
$$

Suppose that $H \equiv 0$. From (3.4) and (3.6) we obtain

$$
\frac{F^{\prime}\left(a^{\prime} G-a G^{\prime}\right)}{(F-1)(G-a)} \equiv \frac{G^{\prime}\left(a^{\prime} F-a F^{\prime}\right)}{(G-1)(F-a)} \text {. }
$$

If $a$ is a constant, noting $a \neq 1$, from (3.10) we get $F \equiv G$, which contradicts (3.4). Thus, $a$ is not a constant. From (3.10) we have

$$
\frac{F^{\prime}\left(a^{\prime} G-a G^{\prime}\right)}{G^{\prime}\left(a^{\prime} F-a F^{\prime}\right)}-1 \equiv \frac{(F-1)(G-a)}{(G-1)(F-a)}-1 .
$$

Thus,

$$
\frac{a^{\prime}\left[\left(F^{\prime}-G^{\prime}\right) G-(F-G) G^{\prime}\right]}{G^{\prime}\left(a^{\prime} F-a F^{\prime}\right)} \equiv \frac{(1-a)(F-G)}{(G-1)(F-a)} .
$$


From this we get

$$
\frac{F^{\prime}-G^{\prime}}{F-G} \equiv \frac{(1-a) G^{\prime}\left(a^{\prime} F-a F^{\prime}\right)}{a^{\prime} G(G-1)(F-a)}+\frac{G^{\prime}}{G}
$$

By (3.5), we know that there is a point $z_{0}$ such that $z_{0}$ is a common zero of $F-b_{5}$ and $G-b_{5}$ that is not a zero or a pole of $a, a^{\prime}, b_{5}, b_{5}-1, b_{5}-a$. It is obvious that $z_{0}$ is a pole of the left-hand side of (3.11), and not a pole of the right-hand side of (3.11), which is a contradiction. Thus,

$$
H \not \equiv 0 .
$$

Suppose that $z_{n}$ is a common zero of $F-b_{5}$ and $G-b_{5}$ that is not a zero or pole of $a, b_{5}, b_{5}-1, b_{5}-a$. It is obvious that $z_{n}$ is a zero of $F-G$, and $z_{n}$ is not a pole of

$$
\frac{Q}{F(F-1)(F-a) G(G-1)(G-a)},
$$

where $Q$ is given by (3.8). By (3.7), we know that $z_{n}$ is a zero of $H$. Again by (3.12) we obtain

$$
\begin{aligned}
\bar{N}_{0}\left(r, b_{5}, F, G\right) & \leq N(r, 0, H)+S(r, F)+S(r, G) \\
& \leq m(r, H)+N(r, H)+S(r, F)+S(r, G) .
\end{aligned}
$$

From (3.6) we have

$$
\begin{aligned}
H= & \frac{F^{\prime}}{F-1} \cdot \frac{a^{\prime} G-a G^{\prime}}{G(G-a)}-\left(\frac{F^{\prime}}{F-1}-\frac{F^{\prime}}{F}\right) \cdot \frac{a^{\prime} G-a G^{\prime}}{G-a} \\
& -\left(\frac{G^{\prime}}{G-1}-\frac{G^{\prime}}{G}\right) \cdot \frac{a^{\prime} F-a F^{\prime}}{F-a}+\frac{G^{\prime}}{G-1} \cdot \frac{a^{\prime} F-a F^{\prime}}{F(F-a)} .
\end{aligned}
$$

Again by Lemma 3 and the lemma of the logarithmic derivative we obtain

$$
m(r, H)=S(r, F)+S(r, G) .
$$

Substituting (3.15) into (3.13) we have

$$
\bar{N}_{0}\left(r, b_{5}, F, G\right) \leq N(r, H)+S(r, F)+S(r, G) .
$$

Next we estimate on $N(r, H)$.

By (3.6), we know that the poles of $H$ only possibly occur from the zeros of $F$, $G, F-1, G-1, F-a$ and $G-a$, the poles of $F, G$ and $a$. Let $S_{0}$ be the set of all zeros, 1-points and poles of $a$, and let for $j=1,2,3,4$

$$
A_{j}:=\left\{z \mid F(z)-b_{j}(z)=0\right\} \backslash S_{0}, \quad B_{j}:=\left\{z \mid G(z)-b_{j}(z)=0\right\} \backslash S_{0},
$$

where $b_{1}=0, b_{2}=\infty, b_{3}=1$ and $b_{4}=a$. Thus, the poles of $H$ occur possibly only from the set

$$
\bigcup_{1 \leq p \leq 4} A_{p} \bigcup_{1 \leq q \leq 4} B_{q} \bigcup S_{0}
$$


Let

$$
\begin{aligned}
S_{1}: & =\bigcup_{1 \leq p \leq 4}\left\{A_{p} \bigcap B_{p}\right\}, \\
S_{2} & :=\left\{\bigcup_{1 \leq p \leq 4} A_{p}\right\} \backslash\left\{\bigcup_{1 \leq q \leq 4} B_{q}\right\}, \\
S_{3}:= & \left\{\bigcup_{1 \leq q \leq 4} B_{q}\right\} \backslash\left\{\bigcup_{1 \leq p \leq 4} A_{p}\right\}, \\
S_{4}:= & \bigcup_{\substack{1 \leq p \leq 4 \\
1 \leq q \leq 4 \\
p \neq q}}\left\{A_{p} \bigcap B_{q}\right\} .
\end{aligned}
$$

It is clear that $S_{1}$ is a set of the common zeros of $F-b_{j}$ and $G-b_{j} \quad(j=1,2,3,4)$; $S_{2}$ is a set of the zeros of $F-b_{j} \quad(j=1,2,3,4)$ that is not the zeros of $G-b_{k}$ $(k=1,2,3,4) ; S_{3}$ is a set of the zeros of $G-b_{j} \quad(j=1,2,3,4)$ that is not the zeros of $F-b_{k} \quad(k=1,2,3,4) ; S_{4}$ is a set of the zeros of $F-b_{j}$ that is the zeros of $G-b_{k}$, where $1 \leq j, k \leq 4$ and $j \neq k$. From this we have

$$
\bigcup_{1 \leq j \leq 4} S_{j}=\bigcup_{1 \leq p \leq 4} A_{p} \bigcup_{1 \leq q \leq 4} B_{q}
$$

Thus, the poles of $H$ occur possibly only from the set

$$
\bigcup_{1 \leq j \leq 4} S_{j} \bigcup S_{0}
$$

Since $b_{1}, b_{2}, b_{3}$ and $b_{4}$ are four distinct elements in $\{S(F) \cap S(G)\} \cup\{\infty\}$, the contribution of $S_{0}$ to $N(r, H)$ is at most $S(r, F)+S(r, G)$. We next estimate the contribution of $\bigcup_{1 \leq j \leq 4} S_{j}$ to $N(r, H)$. We discuss four cases:

Case 1. The contribution of $S_{1}$ to $N(r, H)$.

We distinguish four subcases:

Subcase 1.1. Suppose that $z_{11} \in A_{1} \cap B_{1}$, and assume that $z_{11}$ is a zero of $F$ of order $p_{1}$ and $G$ of order $q_{1}$. Then from (3.9), we know that $z_{11}$ is a zero of $Q$ of order at least $p_{1}+q_{1}-1$. Noting that $z_{11}$ is a zero of $F-G$, from (3.7) we deduce that $z_{11}$ is not a pole of $H$.

Subcase 1.2. Suppose that $z_{12} \in A_{2} \cap B_{2}$, and assume that $z_{12}$ is a pole of $F$ of order $p_{2}$ and $G$ of order $q_{2}$. From (3.9), we know that $z_{12}$ is a pole of $Q$ of order at most $2 p_{2}+2 q_{2}+1$. Noting that $z_{12}$ is a pole of $F-G$ of order at most $\max \left\{p_{2}, q_{2}\right\}$, from (3.7), we have that $z_{12}$ is not a pole of $H$.

Subcase 1.3. Suppose that $z_{13} \in A_{3} \cap B_{3}$. Noting that $z_{13}$ is a zero of $F-G$, a simple pole of $\frac{F^{\prime}}{F-1}$ and $\frac{G^{\prime}}{G-1}$, from (3.6) we have that $z_{13}$ is not a pole of $H$.

Subcase 1.4. Suppose that $z_{14} \in A_{4} \cap B_{4}$. From (2.6), we know that $z_{14}$ is a simple pole of $\frac{a^{\prime} F-a F^{\prime}}{F(F-a)}$ and $\frac{a^{\prime} G-a G^{\prime}}{G(G-a)}$. Noting that $z_{14}$ is a zero of $F-G$, from (3.6) we see that $z_{14}$ is not a pole of $H$.

From the above, it follows that the points in $S_{1}$ are not poles of $H$. Thus, the contribution of $S_{1}$ to $N(r, H)$ is 0 . 
Case 2. The contribution of $S_{2}$ to $N(r, H)$.

We distinguish four subcases:

Subcase 2.1. Suppose that $z_{21} \in A_{1}$ and $z_{21} \notin \bigcup_{1 \leq q \leq 4} B_{q}$. Then $z_{21}$ is a zero of $F$, not a zero of $G, 1 / G, G-1$ and $G-a$. From (3.6), we have that $z_{21}$ is a pole of $H$ of order at most 1 .

Subcase 2.2. Suppose that $z_{22} \in A_{2}$ and $z_{22} \notin \bigcup_{1<q<4} B_{q}$. Then $z_{22}$ is a pole of $F$, not a zero of $G, 1 / G, G-1$ and $G-a$. From (3.6), we have that $z_{22}$ is a pole of $H$ of order at most 1 .

Subcase 2.3. Suppose that $z_{23} \in A_{3}$ and $z_{23} \notin \bigcup_{1<q<4} B_{q}$. Then $z_{23}$ is a zero of $F-1$, not a zero of $G, 1 / G, G-1$ and $G-a$. From (3.6), we have that $z_{23}$ is a pole of $H$ of order at most 1 .

Subcase 2.4. Suppose that $z_{24} \in A_{4}$ and $z_{24} \notin \bigcup_{1 \leq q \leq 4} B_{q}$. Then $z_{24}$ is a zero of $F-a$, not a zero of $G, 1 / G, G-1$ and $G-a$. From (2.6) and (3.6), we have that $z_{24}$ is a pole of $H$ of order at most 1 . 1 .

From the above, we know that the points in $S_{2}$ are poles of $H$ of order at most

Case 3. The contribution of $S_{3}$ to $N(r, H)$.

As with Case 2, we have that the points in $S_{3}$ are poles of $H$ of order at most 1 .

Case 4. The contribution of $S_{4}$ to $N(r, H)$.

Suppose that $z_{4} \in S_{4}$. Then $z_{4} \in A_{p}$ and $z_{4} \in B_{q}$, where $1 \leq p, q \leq 4$ and $p \neq q$. Without loss of generality we can assume that $z_{4} \in A_{1}$ and $z_{4} \in B_{2}$. Then, $z_{4}$ is a zero of $F$, and a pole of $G$. From (2.6) and (3.6), we have that $z_{4}$ is a pole of $H$ of order at most 2. Thus, the points in $S_{4}$ are poles of $H$ of order at most 2.

Noting that each point of $S_{2}$ and $S_{3}$ is counted once, each point of $S_{4}$ is counted twice in

$$
\sum_{j=1}^{4} \bar{N}_{12}\left(r, b_{j}, F, G\right)
$$

From the above and (1.1) we obtain that the contribution of $\bigcup_{1 \leq j \leq 4} S_{j}$ to $N(r, H)$ is at most

$$
\sum_{j=1}^{4} \bar{N}_{12}\left(r, b_{j}, F, G\right)
$$

Thus,

$$
N(r, H) \leq \sum_{j=1}^{4} \bar{N}_{12}\left(r, b_{j}, F, G\right)+S(r, F)+S(r, G) .
$$

Substituting (3.17) into (3.16) we have

$$
\bar{N}_{0}\left(r, b_{5}, F, G\right) \leq \sum_{j=1}^{4} \bar{N}_{12}\left(r, b_{j}, F, G\right)+S(r, F)+S(r, G),
$$

i.e.,

$$
\bar{N}_{0}\left(r, a_{5}, f, g\right) \leq \sum_{j=1}^{4} \bar{N}_{12}\left(r, a_{j}, f, g\right)+S(r, f)+S(r, g),
$$

and Lemma 4 is proved. 


\section{THEOREM 2 AND ITS PROOF}

Using Lemma 4, we can prove the following Theorem:

Theorem 2. Let $f$ and $g$ be non-constant meromorphic functions and let $a_{j} \quad(j=$ $1,2, \ldots, 5)$ be five distinct elements in $\{S(f) \cap S(g)\} \cup\{\infty\}$. If $f \not \equiv g$, then

$$
2 T(r, f)+2 T(r, g) \leq 9 \sum_{j=1}^{5} \bar{N}_{12}\left(r, a_{j}, f, g\right)+S(r, f)+S(r, g) .
$$

Proof. By Lemma 4 we have

$$
\bar{N}_{0}\left(r, a_{5}, f, g\right) \leq \sum_{j=1}^{4} \bar{N}_{12}\left(r, a_{j}, f, g\right)+S(r, f)+S(r, g) .
$$

Noting

$$
\bar{N}\left(r, a_{5}, f\right)+\bar{N}\left(r, a_{5}, g\right)=2 \bar{N}_{0}\left(r, a_{5}, f, g\right)+\bar{N}_{12}\left(r, a_{5}, f, g\right),
$$

from this and (4.2) we get

$\bar{N}\left(r, a_{5}, f\right)+\bar{N}\left(r, a_{5}, g\right) \leq 2 \sum_{j=1}^{5} \bar{N}_{12}\left(r, a_{j}, f, g\right)-\bar{N}_{12}\left(r, a_{5}, f, g\right)+S(r, f)+S(r, g)$.

In the same manner as above, we have for $i=1,2,3,4$

$\bar{N}\left(r, a_{i}, f\right)+\bar{N}\left(r, a_{i}, g\right) \leq 2 \sum_{j=1}^{5} \bar{N}_{12}\left(r, a_{j}, f, g\right)-\bar{N}_{12}\left(r, a_{i}, f, g\right)+S(r, f)+S(r, g)$.

By Lemma 1, we have

$$
2 T(r, f)+2 T(r, g) \leq \sum_{i=1}^{5} \bar{N}\left(r, a_{i}, f\right)+\sum_{i=1}^{5} \bar{N}\left(r, a_{i}, g\right)+S(r, f)+S(r, g) .
$$

Substituting (4.3) and (4.4) into (4.5) we obtain (4.1), and Theorem 2 is thus proved.

\section{Proof of Theorem 1}

Suppose that $f \not \equiv g$. By Theorem 2, we can obtain (4.1).

If $k=\infty$, by hypothesis we have $\bar{E}\left(a_{j}, \infty, f\right)=\bar{E}\left(a_{j}, \infty, g\right) \quad(j=1,2, \ldots, 5)$. Thus,

$$
\bar{N}_{12}\left(r, a_{j}, f, g\right)=0 \quad(j=1,2, \ldots, 5) .
$$

Substituting (5.1) into (4.1) we obtain

$$
2 T(r, f)+2 T(r, g)=S(r, f)+S(r, g) .
$$

This is a contradiction. Next, we assume that $k$ is a positive integer.

By hypothesis we have

$$
\bar{E}\left(a_{j}, k, f\right)=\bar{E}\left(a_{j}, k, g\right) \quad(j=1,2, \ldots, 5) .
$$

From this we obtain for $j=1,2, \ldots, 5$

$$
\bar{N}_{12}\left(r, a_{j}, f, g\right) \leq \bar{N}_{(k+1}\left(r, a_{5}, f\right)+\bar{N}_{(k+1}\left(r, a_{5}, g\right)+S(r, f)+S(r, g) .
$$


Substituting (5.2) into (4.1) we obtain

$2 T(r, f)+2 T(r, g) \leq 9 \sum_{j=1}^{5} \bar{N}_{(k+1}\left(r, a_{j}, f\right)+9 \sum_{j=1}^{5} \bar{N}_{(k+1}\left(r, a_{j}, g\right)+S(r, f)+S(r, g)$.

By Lemma 2, we have

$$
\begin{aligned}
& \sum_{j=1}^{5} \bar{N}_{(k+1}\left(r, a_{j}, f\right) \leq \frac{3}{k} T(r, f)+S(r, f), \\
& \sum_{j=1}^{5} \bar{N}_{(k+1}\left(r, a_{j}, g\right) \leq \frac{3}{k} T(r, g)+S(r, g) .
\end{aligned}
$$

Substituting (5.4) and (5.5) into (5.3) we obtain

$$
2 T(r, f)+2 T(r, g) \leq \frac{27}{k} T(r, f)+\frac{27}{k} T(r, g)+S(r, f)+S(r, g),
$$

which contradicts the assumption $k \geq 14$. Thus, $f \equiv g$.

\section{ACKNOWLEDGEMENT}

The author thanks the referee for valuable suggestions and comments.

\section{REFERENCES}

1. W. K. Hayman, Meromorphic Functions, Clarendon Press, Oxford, 1964. MR 29:1337

2. K. Ishizaki and N. Toda, Unicity theorems for meromorphic functions sharing four small functions, Kodai Math. J. 21 (1998), 350-371. MR 99k:30050

3. B. Q. Li, Uniqueness of entire functions sharing four small functions, Amer. J. Math. 119 (1997), 841-858. MR 98m:32001

4. Y. H. Li, Entire functions share four finite small functions IM, Acta Math. Sinica 41 (1998), 249-260. MR 99j:30034

5. Y. H. Li, Meromorphic functions which share four or five small functions, J. Math. Res. Exp. 20 (2000), 94-96.

6. Y. H. Li and J. Y. Qiao, The uniqueness of meromorphic functions concerning small functions, Science in China Ser. (A) 43 (2000), 581-590. MR 2001f:30037

7. R. Nevanlinna, Le théoréme de Picard-Borel et la thèorie des fonctions méromorphes, Gauthier-Villars, Paris, 1929; reprint, Chelsea, 1974. MR 54:5468

8. M. Shirosaki, An extension of unicity theorem for meromorphic functions, Tohoku Math. J. 45 (1993), 491-497. MR 94k:30076

9. N. Toda, Some generalizations of the unicity theorem of Nevanlinna, Proc. Japan Acad. Ser. A Math. Sci. 69 (1993), 61-65. MR 94d:3D056

10. H. X. Yi and C. C. Yang, Uniqueness Theory of Meromorphic Functions, Pure and Applied Math. Monographs No. 32, Science Press, Beijing, 1995.

11. Q. D. Zhang, A uniqueness theorem for meromorphic functions with respect to slowly growing functions, Acta Math. Sinica 36 (1993), 826-833. MR 95f:30047

Department of Mathematics, Shandong University, Jinan 250100, People's Republic OF CHINA

E-mail address: hxyi@sdu.edu.cn 